Classification

Physics Abstracts

$68.00-72.20-73.00$

\title{
INFLUENCE DE LA POLARISATION DU SUBSTRAT SEMI-ISOLANT SUR LES PROPRIÉTÉS ÉLECTRIQUES DU TRANSISTOR A EFFET DE CHAMP A L'ARSÉNIURE DE GALLIUM ET CARACTÉRISATION DE L'INTERFACE ENTRE LA COUCHE ACTIVE ET LE SUBSTRAT SEMI-ISOLANT
}

\author{
P. ROSSEL, H. TRANDUC, J. GRAFFEUIL, C. AZIZI \\ Laboratoire d'Automatique et d'Analyse des Systèmes du C.N.R.S. \\ 7, avenue du Colonel-Roche, 31400 Toulouse, France \\ G. NUZILLAT et G. BERT \\ Laboratoire Central de Recherches Thomson C.S.F. \\ Domaine de Corbeville, 91401 Orsay, France
}

(Reçu le 2 mai 1978, révisé le 3 juillet 1978, accepté le 17 juillet 1978)

\begin{abstract}
Résumé. - Les évolutions du courant de drain et de la capacité de grille d'un transistor à effet de champ à grille métallique sur arséniure de gallium sont étudiées en fonction de la polarisation du substrat. Les résultats obtenus sur des composants élaborés sur une couche épitaxiée, par cracking de composés organométalliques directement sur le susbtrat semi-isolant, sont interprétés en introduisant simultanément une double charge d'espace et une charge fixe positive à l'interface entre la couche $\mathrm{N}$ épitaxiale et le substrat semi-isolant dopé au chrome. On propose des méthodes expérimentales appropriées à la détermination des paramètres technologiques et physiques qui caractérisent à la fois la couche $\mathrm{N}$, l'interface et le substrat. On en conclut que les méthodes classiques de relevé des caractéristiques capacité-tension ne sont pas utilisables, sans ambiguité, pour la détermination du profil de dopage des couches.
\end{abstract}

\begin{abstract}
The effects of the substrate bias on the drain current and on the gate input capacitance of GaAs FET's are studied. The experimental results are explained : it is shown that a dual space charge and a positive fixed charge can develop at the $\mathrm{N}$-epitaxial layer-semi-insulating substrate interface. Experimental methods for determining the technological and physical parameters of the $\mathrm{N}$-layer, the interface and the substrate are proposed. It is deduced that the results obtained by using the classical $C(V)$ profiling methods must be carefully analysed.
\end{abstract}

1. Introduction. - Il est établi que les caractéristiques de l'interface semiconducteur-semi-isolant influencent fortement les propriétés électriques des composants à l'arseniure de gallium réalisés à partir de couches $\mathrm{N}$ épitaxiales sur semi-isolant. En particulier dans les transistors à effet de champ, les phénomènes de relaxation en basses fréquences du courant drain [1], de même que les performances dynamiques et en bruit de fond [2-3] sont liés aux propriétés de l'interface. De plus, il a été rapporté que la valeur du courant de canal [4] et la valeur de la capacité d'entrée du transistor [5] pouvaient dépendre de la polarisation du substrat.

Nous effectuons dans cet article une étude expérimentale de l'effet de la polarisation du substrat sur les caractéristiques courant-tension du transistor à effet de champ et sur les caractéristiques capacitétension de sa diode Schottky de grille. Une analyse phénoménologique, basée d'une part sur l'existence d'une double charge d'espace et d'autre part sur la présence d'une charge fixe, à l'interface semiconducteur-semi-isolant, est proposée. On en déduit de nouvelles méthodes de détermination des paramètres caractéristiques de la couche épitaxiale et de l'interface.

2. Etude expérimentale. - 2:1 StruCtures ÉtuDIÉEs. - Les structures (Fig. 1) étudiées sont semblables à des transistors à effet de champ à l'arséniure de gallium à grille Schottky. La zone active est une couche d'arséniure de gallium de type $\mathrm{N}$, dopée au germanium, réalisée par crackịng de composés 


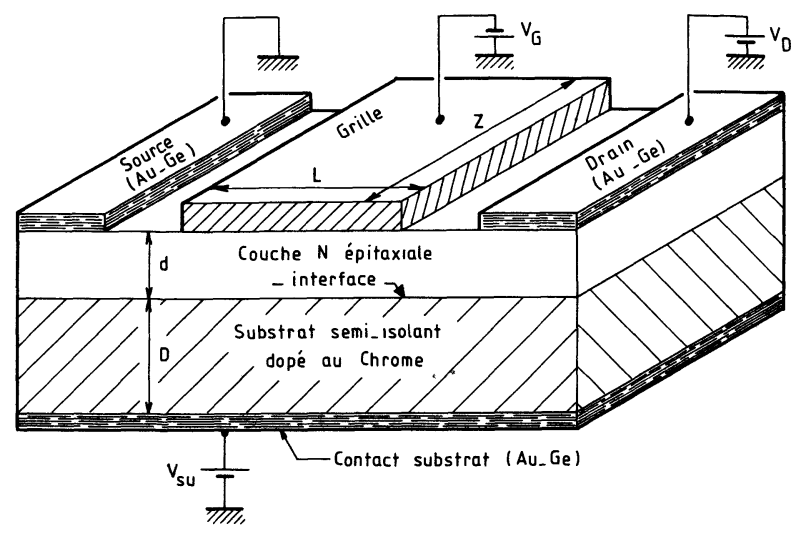

FIG. 1. - Représentation schématique de la structure. Définition des tensions de la polarisation.

[Configuration of the device. Definition of the bias voltages.]

organo-métalliques sous pression réduite [6]. Le dépôt a été effectué directement sur un substrat semi-isolant compensé au chrome, pour lequel le dosage des principales impuretés, effectué par analyse ionique $[16,17]$, a révélé les concentrations suivantes $\left(\mathrm{cm}^{-3}\right)$ :

$\begin{array}{ccccc}\mathrm{Cr} & \mathrm{O} & \mathrm{Fe} & \mathrm{Cu} & \mathrm{Al} \\ 7 \times 10^{16} & 2,5 \times 10^{15} & 2 \times 10^{15} & 9 \times 10^{14} & 1,3 \times 10^{16}\end{array}$

Une triple couche de $\mathrm{Ti} / \mathrm{Pt} / \mathrm{Au}$ déposée sur la couche $\mathrm{N}$ constitue l'électrode de grille dont la forme est rectangulaire. Latéralement, deux contacts ohmiques, obtenus par alliage or-germanium, forment les électrodes de source et de drain. Sur la face arrière du substrat semi-isolant est également réalisé, par alliage, un contact ohmique que nous appellerons l'électrode de substrat. Les dimensions géométriques sont les suivantes :

- l'épaisseur $d$ de la couche $\mathbf{N}$ épitaxiale est comprise entre 0,2 et $0,5 \mu \mathrm{m}$;

- la longueur $L$ dans la direction source-drain, et la largeur $Z$ de la grille sont égales respectivement à $50 \mu \mathrm{m}$ et $300 \mu \mathrm{m}$;

- l'épaisseur $D$ du substrat est voisine de $400 \mu \mathrm{m}$.

2.2 Résultats expérimentaux. - Deux types de mesures ont été effectués, d'une part la mesure de la capacité dynamique entre l'électrode de la grille et l'électrode constituée par la source et le drain réunis, et d'autre part le relevé des caractéristiques en courant entre drain et source. Pour le premier type de mesures les paramètres d'étude sont les tensions continues $V_{G}$ et $V_{\text {su }}$ appliquées respectivement entre grille et source, et substrat et source. La tension drain-source est nulle. Par contre, dans le deuxième cas on fait varier la tension drain-source $V_{\mathbf{D}}$. Quelle que soit la configuration adoptée, la source est toujours prise comme référence des potentiels.

2.2.1 Caractéristiques capacité-tension. - La figure 2 représente les variations de la capacité dyna-

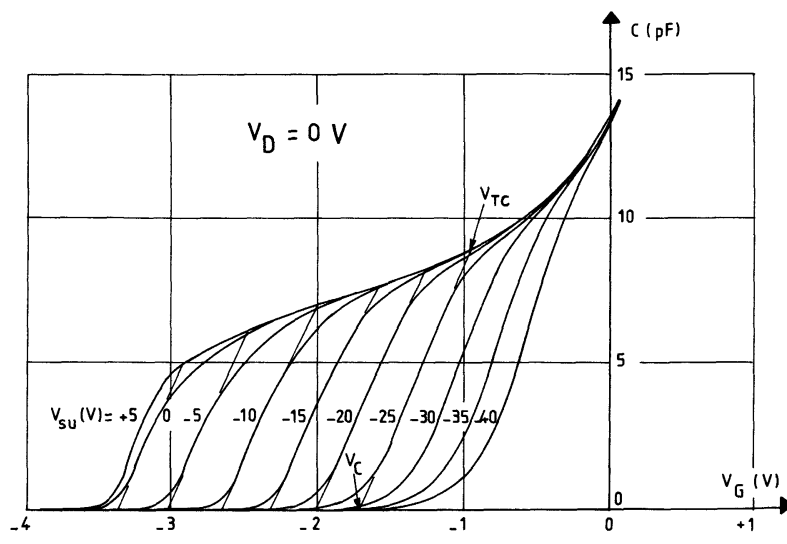

FIG. 2. - Variations expérimentales de la capacité inverse de grille en fonction de la tension de grille pour diverses valeurs de la tension de substrat $V_{\text {su }}$. Définition des tensions $V_{\mathrm{TC}}$ et $V_{\mathrm{C}}$, structure THOMSON SHOT-3, T317 № 1 .

[Experimental variations of the gate capacitance as a function of the gate voltage. The substrate voltage is a parameter.]

mique de grille $C$ en fonction de la tension $V_{\mathrm{G}}$ pour diverses valeurs de la tension $V_{\text {su }}[7,8]$. Sur la caractéristique $C\left(V_{\mathrm{G}}\right)$ on peut définir trois régions (Fig. 2) :

- la première se manifeste par une variation lente de la capacité pour les valeurs de la tension de grille comprises entre 0 et $V_{\mathrm{TC}}$;

- la seconde se caractérise par une chute brusque de cette capacité entre les tensions $V_{\mathrm{TC}}$ et $V_{\mathrm{C}}$;

- la troisième enfin correspond aux tensions inférieures à $V_{\mathrm{C}}$ lorsque la capacité tend vers une valeur nulle.

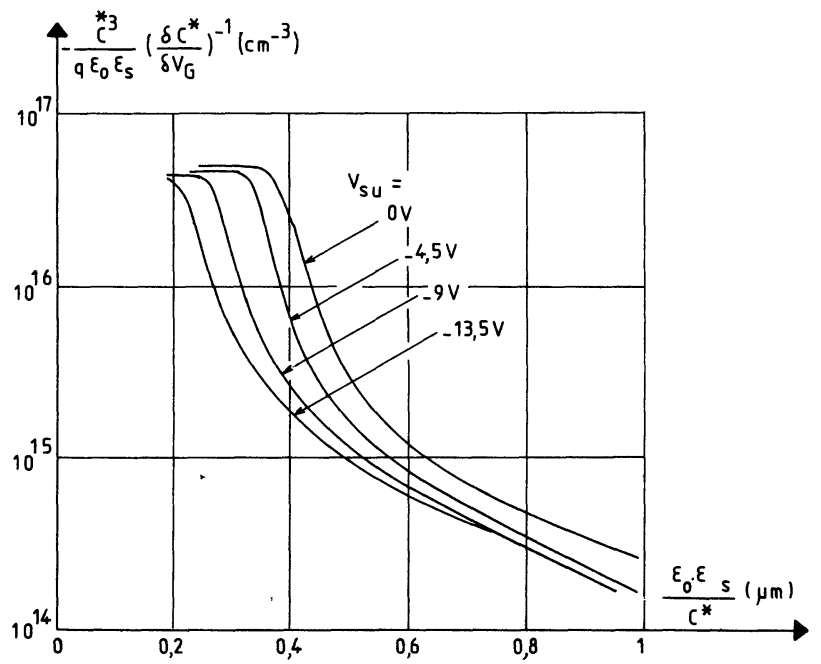

FIG. 3. - Evolutions expérimentales de la quantité $\frac{C^{* 3}}{q \varepsilon_{0} \varepsilon_{\mathrm{s}}}\left(\frac{\partial C^{*}}{\partial V_{\mathrm{G}}}\right)^{-1}$ en fonction de la quantité $\frac{\varepsilon_{0} \varepsilon_{s}}{C^{*}}$ sous diverses valeurs de la tension de substrat $V_{\text {su }}$. Ces caractéristiques ont été relevées par la technique dite de la double goutte de mercure. Couche THOMSON 490-6. [Experimental variations of the quantity $\frac{C^{* 3}}{q \varepsilon_{0} \varepsilon_{\mathrm{s}}}\left(\frac{\partial C^{*}}{\partial V_{\mathrm{G}}}\right)^{-1}$ as a function of the quantity $\frac{\varepsilon_{0} \varepsilon_{\mathrm{s}}}{C^{*}}$. Substrate voltage as parameter (These characteristics are obtained by the two mercury diodes method).] 
Remarquons que les tensions $V_{\mathrm{TC}}$ et $V_{\mathrm{C}}$ dépendent de la polarisation négative du substrat tandis que, au contraire, les valeurs de capacités pour des tensions grille proches de zéro sont peu sensibles à l'effet de polarisation du substrat.

Nous avons tracé en figure 3 les variations de la quantité $-\frac{C^{* 3}}{q \varepsilon_{0} \varepsilon_{\mathrm{s}}}\left(\frac{\partial C^{*}}{\partial V_{\mathrm{G}}}\right)^{-1}$ en fonction de la variable $\varepsilon_{0} \varepsilon_{\mathrm{s}} / C^{*}$, où $\varepsilon_{0} \varepsilon_{\mathrm{s}}$ est la constante diélectrique de l'arséniure de gallium, $q$ là charge de l'électron et $C^{*}$ et la capacité $C$ ramenée à l'unité de surface. Il est généralement admis que cette courbe représente le profil de dopage apparent de la couche $[9,10]$. Or il apparaît ici qu'à chaque valeur de la tension $V_{\text {su }}$ correspond une courbe différente.

2.2.2 Caractéristiques courant-tension. - La figure 4 représente les variations du courant de drain $I_{\mathrm{D}}$ en fonction de la tension de grille, à faible tension drain-source. En fonction de la tension

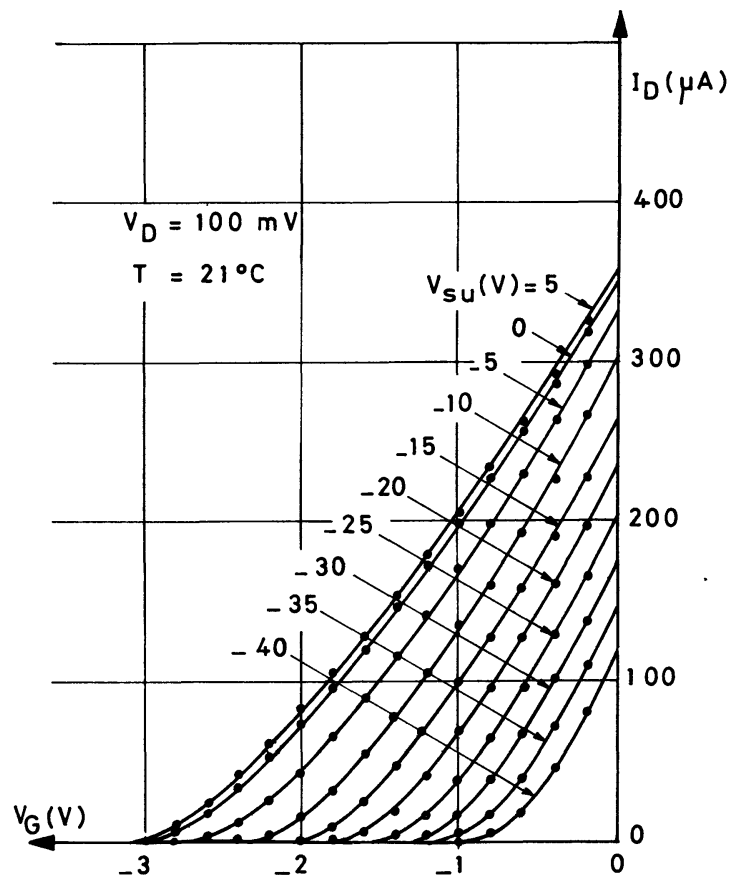

FIG. 4. - Variations expérimentales du courant de drain $I_{\mathrm{D}}$ en fonction de la tension de grille $V_{G}$, pour diverses valeurs de la tension de substrat $V_{\text {su. }}$. Structure THOMSON SCHOT-3, T317 No 2.

[Experimental variations of the drain current as a function of the gate voltage. The substrate voltage is a parameter.]

substrat-source, considérée comme paramètre, la caractéristique $I_{\mathrm{D}}\left(V_{\mathrm{G}}\right)$ se translate suivant l'axe vertical. Plus la tension $V_{\text {su }}$ est négative, plus le courant, à tension grille constante, est faible. En particulier, les variations du courant $I_{\mathrm{DO}}$, correspondant à la tension grille nulle, en fonction de la tension substratsource sont représentées en figure 5. Pour les valeurs positives ou légèrement négatives de $V_{\text {su }}$, le courant $I_{\mathrm{DO}}$ reste constant à une valeur que nous noterons $I_{\mathrm{DL}}$. Puis la tension $V_{\text {su }}$ dépassant en valeurs négatives

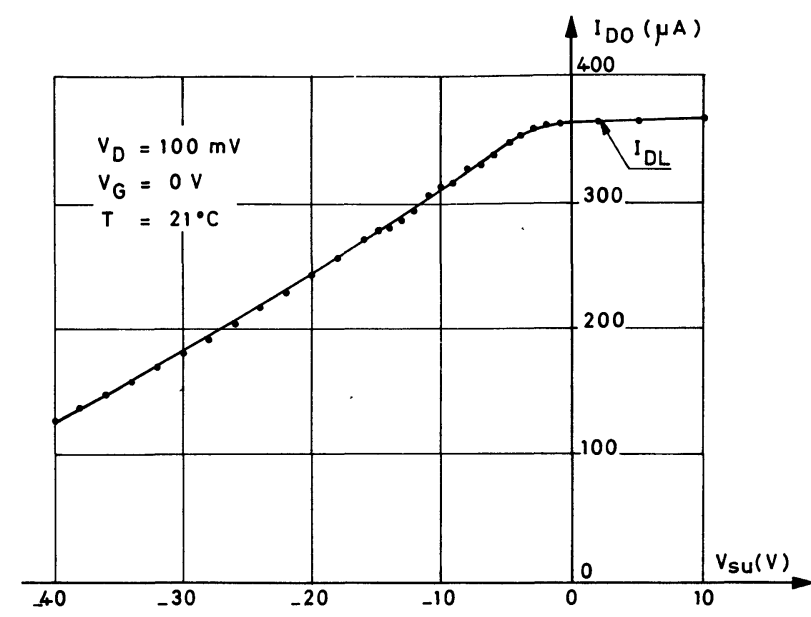

Fig. 5. - Variations expérimentales du courant $I_{\text {Do }}$ à tension grille-source nulle $\left(V_{\mathrm{G}}=0\right)$ en fonction de la tension de substrat $V_{\mathrm{su}}$. Structure THOMSON SCHOT-3, T317 № 2.

[Experimental variations of the drain current, at $V_{G}=0$, as a function of the substrate voltage.]

un seuil que nous noterons $V_{\text {sul }}$, le courant $I_{\mathrm{DO}}$ diminue lorsque $V_{\text {su }}$ augmente en valeur absolue. Remarquons que $I_{\mathrm{Do}}$ évolue très peu pour les tensions $V_{\text {su }}$ positives ou légèrement négatives. Ce fait est à rapprocher de la faible modification de la courbe de capacité $C\left(V_{\mathrm{G}}\right)$ dans les mêmes conditions de polarisation du substrat.

La figure 6 représente enfin les caractéristiques de sortie $I_{\mathrm{D}}\left(V_{\mathrm{D}}\right)$ paramétrées en tension grille $V_{\mathrm{G}}$, pour deux valeurs particulières de la tension de substrat 0 et $-40 \mathrm{~V}$. On constate que non seulement la valeur moyenne du courant diminue, mais que, de plus,

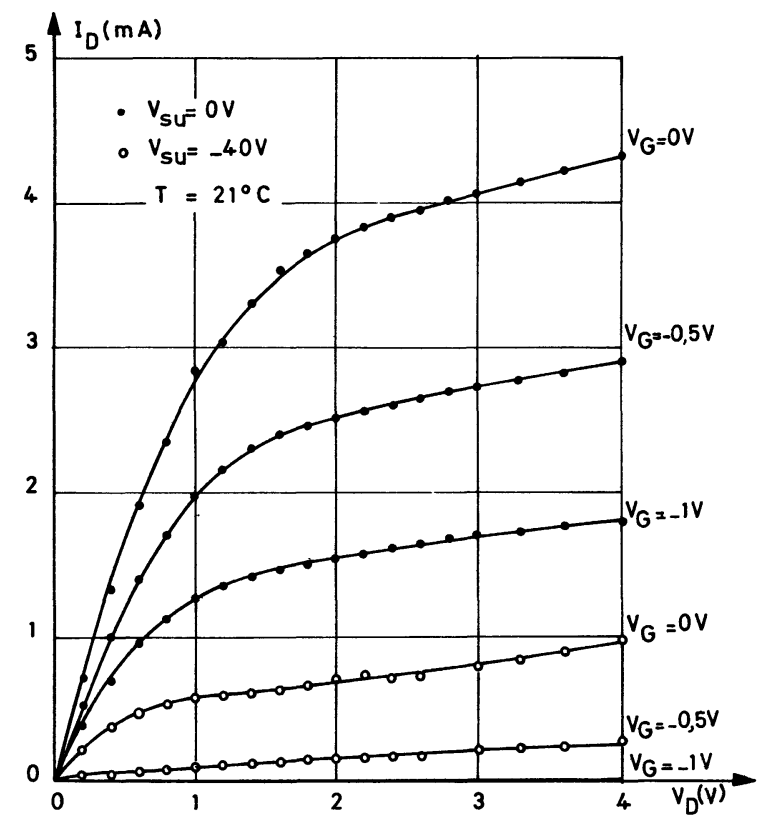

Fig. 6. - Variations expérimentales du courant de drain $I_{D}$ en fonction de la tension de drain $V_{\mathrm{D}}$, pour diverses valeurs des tensions de grille $V_{\mathrm{G}}$ et de substrat $V_{\mathrm{su}}$. Structure THOMSON SCHOT-3, T317 No 2.

[Experimental variations of the drain current as a function of the drain voltage. Gate and substrate voltages are parameters.] 

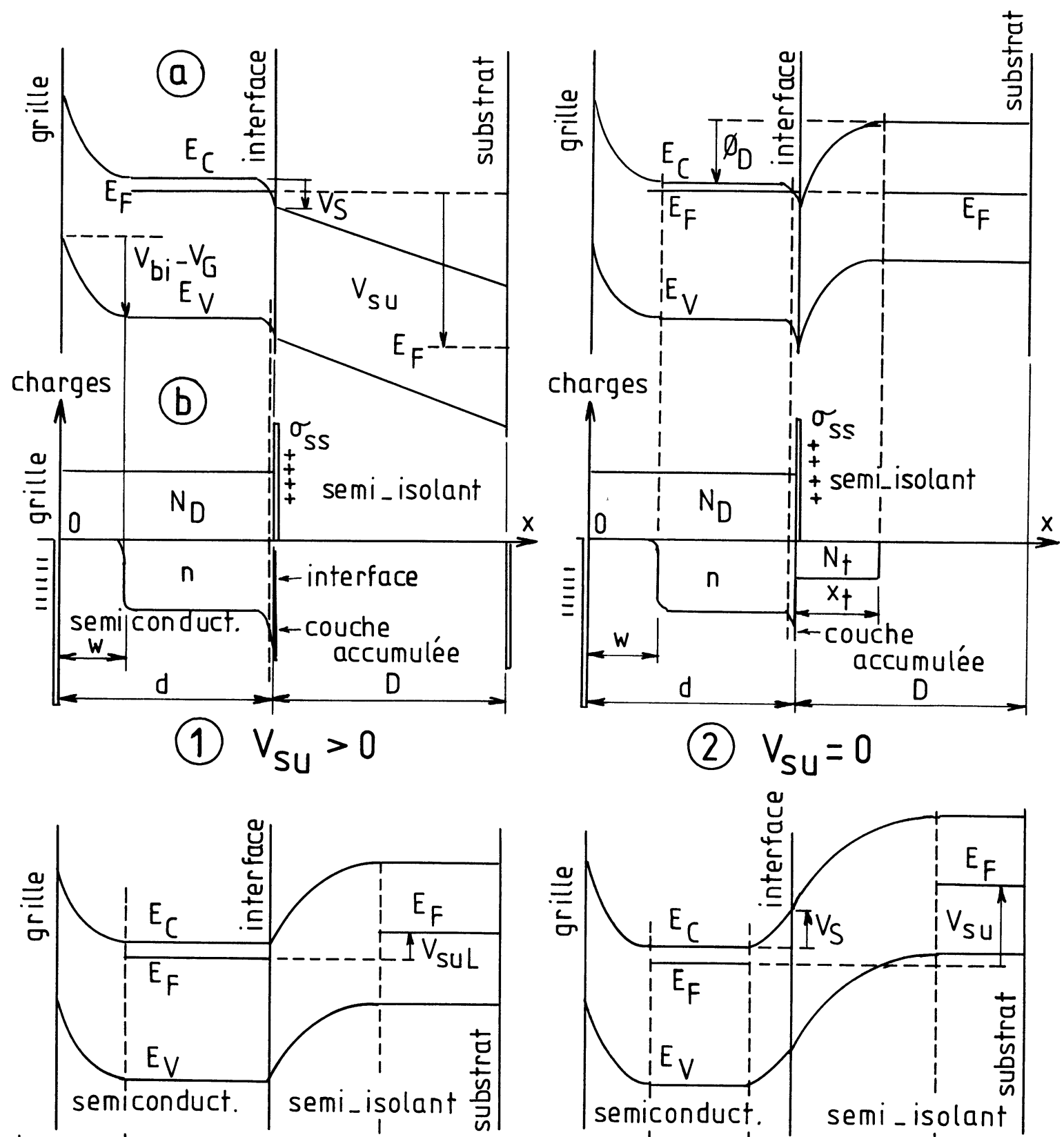

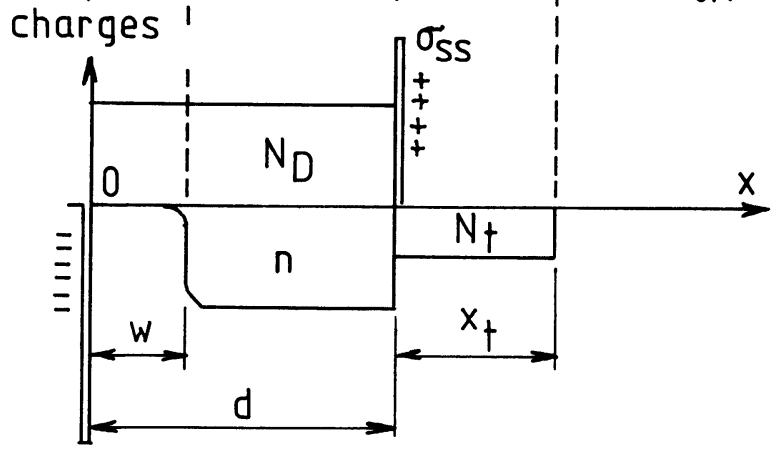

(3) $v_{S U}=V_{S U L}$

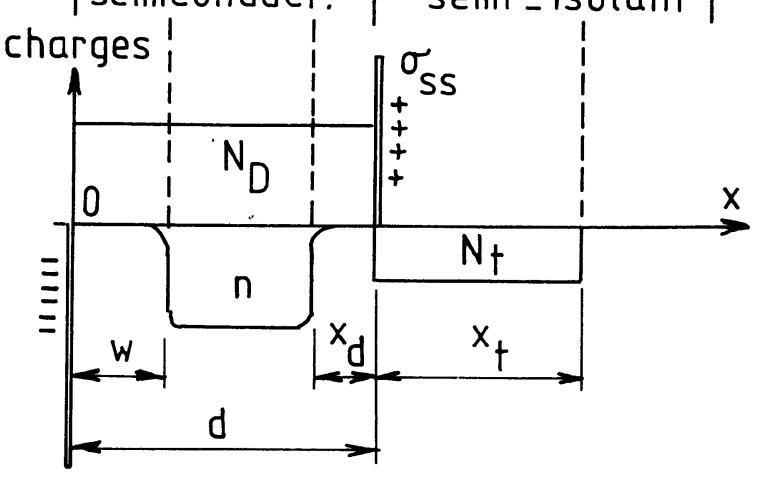

(4) $v_{S U}<V_{S U L}$

Fig. 7. - Variations du potentiel électrostatique et des charges dans une coupe transversale de la structure.

[Electrostatic potential and charge configurations in a cross-section of the structure.] 
la tension de saturation $V_{\mathrm{D} \text { sat }}$ (transition entre la zone ohmique et la zone saturée) diminue lorsque le potentiel $V_{\text {su }}$ augmente négativement.

3. Analyse qualitative. - Lorsqu'une différence de potentiel $V_{\text {su }}$ est appliquée entre le fond du substrat semi-isolant et la couche épitaxiale portée au potentiel de référence, il se produit une répartition de ce potentiel entre la couche épitaxiale et le semi-isolant. On désignera par $V_{\mathrm{s}}$ la valeur du potentiel à l'interface semiconducteur-semi-isolant. Corrélativement, des zones de charges d'espace se développent au sein de la structure afin d'assurer sa neutralité électrique. La nature et la forme de ces charges d'espace dépendent de la polarité des tensions et de leurs amplitudes.

Compte tenu des propriétés que nous avons observées expérimentalement, des résultats rapportés et des hypothèses émises par les différents auteurs qui ont étudié à la fois les phénomènes de relaxation en très basses fréquences dans les couches épitaxiales d'arséniure de gallium $[1,11]$ et les propriétés de l'interface semiconducteur-semi-isolant [4, 12], les mécanismes que nous retiendrons pour décrire qualitativement les modifications des caractéristiques capacité-tension et courant-tension, sont les suivants :

(i) sous l'électrode de grille, il se crée une région de charge d'espace dépeuplée, de largeur $W$, sous l'effet de la polarisation inverse grille-source ;

(ii) à l'interface semiconducteur-semi-isolant, il existe une charge fixe superficielle, analogue à celle que l'on a pu observer dans les interfaces semiconducteur-isolant. Cette charge sera considérée comme positive et sa valeur par unité de surface sera notée $\sigma_{\text {ss }}$;

(iii) de part et d'autre de cet interface, des charges d'espace doivent apparaître pour satisfaire la loi de continuité de l'induction électrique. En particulier, selon l'hypothèse que nous avons émise [1] sur la possibilité de formation d'une charge négative dans le semi-isolant, cette charge peut être associée à des centres profonds en densité $N_{\mathrm{t}}$. L'origine physique de ces centres peut être attribuée [18] aux impuretés qui contribuent à rendre semi-isolant le substrat. Leur niveau d'énergie, dans les conditions de bande plate, se situe au-dessus du niveau de Fermi à quelques unités thermodynamiques $(k T)$.

Sur la base de ces hypothèses, les configurations de potentiel et de charge dans la structure, à tension drain-source nulle, sont les suivantes :

(i) la tension $V_{\text {su }}$ est positive : la structure se comporte comme une structure semiconducteurisolant dont le diagramme de bandes d'énergie correspond à celui de la figure 7.1a. Le semiconducteur est accumulé en électrons du côté de l'interface et est dépeuplé sous la grille (Fig. 7.1b). Les variations du potentiel dans le semi-isolant sont linéaires;

(ii) la tension $V_{\text {su }}$ est nulle : il y a formation d'une charge d'espace négative associée aux centres $N_{\mathrm{t}}$ dans le semi-isolant, et le semiconducteur est encore accumulé à l'interface. Dans le corps du semi-isolant, loin de l'interface, le potentiel est constant (Fig. 7.2) ;

(iii) la tension de substrat est négative et égale à la valeur $V_{\text {sul }}$ : les bandes d'énergie restent horizontales dans le semiconducteur et ce dernier est neutre près de l'interface avec le semi-isolant. La charge négative dans le semi-isolant augmente (Fig. 7.3);

(iii) la tẹnsion de substrat est inférieure à la valeur $V_{\text {sul }}$ : le semiconducteur devient dépeuplé près de l'interface et une charge positive s'y développe sur une distance $x_{\mathrm{d}}$ comptée à partir de cet interface. Dans le semi-isolant, la charge négative s'étend vers le fond du substrat (Fig. 7.4).

Ainsi, et en négligeant en première approximation les effets d'accumulation de porteurs qui se produisent pour des tensions $V_{\text {su }}$ supérieures à $V_{\text {sul }}$, il apparaît que ce n'est que dans le cas de tension de substrat inférieure à $V_{\text {sul }}$ que la charge totale des porteurs majoritaires dans la couche semiconductrice est fortement modulée par la polarisation de substrat. Par suite, la résistance, associée à ces porteurs, vue entre drain et source, augmente lorsque $V_{\text {su }}$ est de plus en plus négatif.

D'autre part, la capacité de grille, associée à la modulation de la largeur $W$ de la région dépeuplée sous la grille, ne dépend que du profil de dopage tant que cette distance $W$ reste inférieure à la quantité $d-x_{\mathrm{d}}$.

Lorsque les deux charges d'espace associées aux distances $W$ et $x_{\mathrm{d}}$ se rejoignent de sorte que, en tout point du semiconducteur, la densité des électrons devient inférieure à la densité $N_{\mathrm{D}}$, le courant entre source et drain diminue rapidement et la capacité dynamique vue de la grille tend vers une valeur nulle.

Dans ce cas la répartition en électrons est Gaussienne avec un écart-type voisin de la longueur de Debye extrinsèque [13]. La chute rapide de capacité n'est plus l'image du profil de dopage et, le faible courant, que l'on peut observer entre drain et source, est associé à la transition entre deux mécanismes de conduction [14].

D'un point de vue pratique, on idéalise la situation en supposant que les charges d'espace désertées possèdent une frontière abrupte, et en définissant la limite d'interaction par l'existence d'un point unique du semiconducteur présentant une densité $n$ égale à $N_{\mathrm{D}}$. La tension correspondante est appelée tension de seuil $V_{\mathrm{T}}$. Elle est voisine de la tension $V_{\mathrm{TC}}$ définie précédemment.

4. Analyse quantitative. - Le but de l'analyse quantitative est d'établir les relations liant courant et capacité de grille aux tensions grille-source et substrat-source. Les principales hypothèses qui seront utilisées sont :

- les densités effectives de dopant $N_{\mathrm{D}}$ dans le semiconducteur et de centres profonds $N_{\mathrm{t}}$ dans le semi-isolant sont uniformes en distance; 
- les charges d'espace désertées seront supposées abruptes, c'est-à-dire qu'on négligera les effets de distribution d'électrons dans les fronts de ces charges d'espace.

Par ailleurs on ne traitera de façon approfondie que le cas où la tension de substrat est négative.

\subsection{ETUde THÉORIQUe. - 4.1.1 Expressions de} $x_{\mathrm{d}}$ et $V_{\text {sul }}$. - Lorsque la tension de substrat est négative $\left(V_{\text {su }}<V_{\text {sul }}\right)$, il apparaît deux charges d'espace à l'interface, positive dans le semiconducteur et négative dans le semi-isolant. La charge positive est due aux impuretés ionisées $\left(N_{\mathrm{D}}\right)$, sur une distance $x_{\mathrm{d}}$, et la charge négative est associée aux centres $\left(N_{t}\right)$ qui peuvent s'ioniser sur une distance $x_{t}$ (Fig. 7.4). La relation de continuité de l'induction électrique normale est :

$$
q N_{\mathrm{D}} x_{\mathrm{d}}=q N_{\mathrm{t}} x_{\mathrm{t}}-\sigma_{\mathrm{ss}}
$$

soit encore, après intégration de l'équation de Poisson dans le semiconducteur et le semi-isolant :

$$
\begin{aligned}
& \sqrt{2 \varepsilon_{0} \varepsilon_{\mathrm{s}} q N_{\mathrm{D}}\left(-V_{\mathrm{s}}\right)}= \\
& \quad=\sqrt{2 \varepsilon_{0} \varepsilon_{\mathrm{s}} q N_{\mathrm{t}}\left(\varphi_{\mathrm{D}}-V_{\mathrm{su}}+V_{\mathrm{s}}\right)}-\sigma_{\mathrm{ss}}
\end{aligned}
$$

où $\varphi_{\mathrm{D}}$ est la tension de diffusion. Par suite, la distance $x_{\mathrm{d}}$ peut être approximée, dans le cas où la densité $N_{\mathrm{t}}$ est très inférieure au dopage $N_{\mathrm{D}}$, par :

$$
x_{\mathrm{d}} \simeq \sqrt{\frac{2 \varepsilon_{0} \varepsilon_{\mathrm{s}}}{q N_{\mathrm{D}}} \frac{N_{\mathrm{t}}}{N_{\mathrm{D}}}\left(\varphi_{\mathrm{D}}-V_{\mathrm{su}}\right)}-\frac{\sigma_{\mathrm{ss}}}{q N_{\mathrm{D}}} .
$$

Cette relation fournit la condition d'existence de la zone dépeuplée dans le semiconducteur à l'interface :

$$
V_{\text {su }} \leqslant V_{\text {sul }}
$$

avec

$$
V_{\text {sul }}=\varphi_{\mathrm{D}}-\frac{\sigma_{\mathrm{ss}}^{2}}{2 \varepsilon_{0} \varepsilon_{\mathrm{s}} q N_{\mathrm{t}}}
$$

4.1.2 Expression du courant drain à faible tension drain-source. - La tension appliquée en inverse sur la grille dépeuple le semiconducteur sur une distance

$$
W=\sqrt{\frac{2 \varepsilon_{0} \varepsilon_{\mathrm{s}}}{q N_{\mathrm{D}}} \cdot\left(V_{\mathrm{bi}}-V_{\mathrm{G}}\right)}
$$

$V_{\mathrm{bi}}$ est la tension de diffusion de la diode Schottky de grille.

L'épaisseur effective du canal de conduction peuplé d'électrons est égale à $d-\left(W+x_{d}\right)$ (Fig. 8). Par suite le courant drain du transistor, à faible tension drain-source, s'obtient par la méthodologie classique. En négligeant les résistances d'accès de source et de drain devant la résistance de canal (cas des

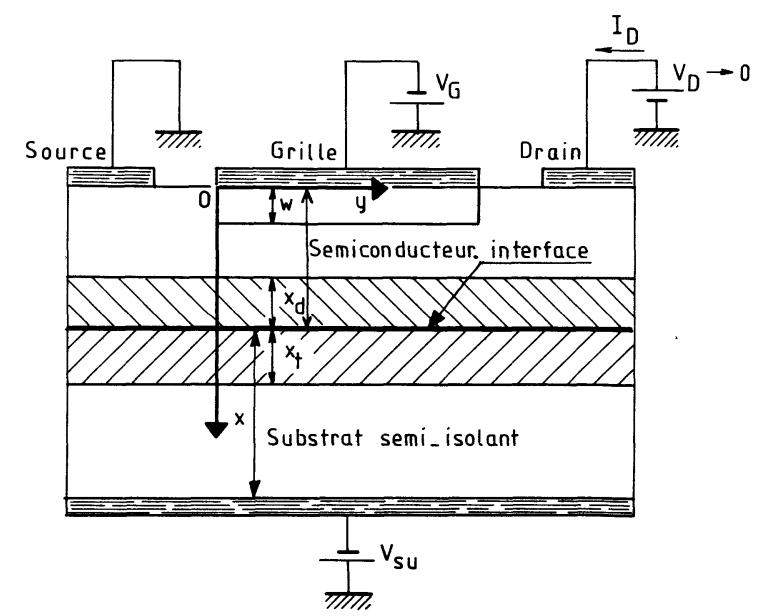

FIG. 8. - Définition des diverses épaisseurs $W, x_{\mathrm{d}}$ et $x_{\mathrm{t}}$ et des coordonnées $x$ et $y$ lorsque $V_{\mathrm{D}}$ tend vers zéro.

[Definitions of $W, x_{\mathrm{d}}, x_{\mathrm{t}}$ thicknesses and the $x$ and $y$ coordinates.]

transistors à grille longue), l'expression du courant est :

a) si $V_{\text {su }}>V_{\text {sul }}$

$$
I_{\mathrm{D}}=\mu_{0} \frac{Z}{L} q N_{\mathrm{D}} V_{\mathrm{D}}\left[d\left(1-\sqrt{\frac{V_{\mathrm{bi}}-V_{\mathrm{G}}}{V_{\mathrm{bi}}-V_{\mathrm{T}}}}\right)\right]
$$

où $\mu_{0}$ est la mobilité à champ faible et $V_{\mathrm{T}}$ la tension définie par :

$$
V_{\mathrm{T}}=V_{\mathrm{bi}}-\frac{q N_{\mathrm{D}} d^{2}}{2 \varepsilon_{0} \varepsilon_{\mathrm{s}}} .
$$

b) Si $V_{\text {su }} \leqslant V_{\text {sul }}$

$$
I_{\mathrm{D}}=\mu_{0} \frac{Z}{L} q N_{\mathrm{D}} V_{\mathrm{D}}\left[\left(d-x_{\mathrm{d}}\right)\left(1-\sqrt{\frac{V_{\mathrm{bi}}-V_{\mathrm{G}}}{V_{\mathrm{bi}}-V_{\mathrm{T}}}}\right)\right]
$$

la tension $V_{\mathrm{T}}$ étant alors définie par :

avec

$$
V_{\mathrm{T}}=V_{\mathrm{bi}}-\frac{q N_{\mathrm{D}}\left(d-x_{\mathrm{d}}\right)^{2}}{2 \varepsilon_{0} \varepsilon_{\mathrm{s}}}
$$

$$
x_{\mathrm{d}} \simeq \sqrt{\frac{2 \varepsilon_{0} \varepsilon_{\mathrm{s}}}{q N_{\mathrm{D}}^{2}} N_{\mathrm{t}}\left(\varphi_{\mathrm{D}}-V_{\mathrm{su}}\right)}-\frac{\sigma_{\mathrm{ss}}}{q N_{\mathrm{D}}} .
$$

Par ailleurs, sur la base des hypothèses de fronts de charges d'espace abrupts, le courant s'annule théoriquement pour :

$$
V_{\mathrm{G}}=V_{\mathrm{T}}
$$

Les tensions de seuil $V_{\mathrm{T}}$ s'expriment respectivement par (8) et (10).

4.1.3 Expression de la capacité dynamique de grille. - Tant que la tension de grille est supérieure à la tension de seuil, la capacité est celle d'une diode Schottky polarisée en inverse :

$$
C=Z L \sqrt{\frac{q \varepsilon_{0} \varepsilon_{\mathrm{s}} N_{\mathrm{D}}}{2\left(V_{\mathrm{bi}}-V_{\mathrm{G}}\right)}} .
$$


Dès que la tension de seuil est atteinte, d'une part la capacité est associée à la modulation de la charge électronique résiduelle dans les charges d'espace dépeuplées et d'autre part, peut être affectée par l'effet de résistance distribuée entre tout point du semiconducteur et les contacts de source et de drain. On vérifie que, dans les conditions expérimentales adoptées, c'est le premier effet qui est prépondérant et qui explique que la capacité chute rapidement entre la tension $V_{\mathrm{TC}}$ et une tension $V_{\mathrm{C}}$. En basses fréquences, un ordre de grandeur de l'écart entre ces deux tensions est :

$$
\left.\left|V_{\mathrm{C}}-V_{\mathrm{T}}\right| \simeq \frac{2 L_{\mathrm{D}}}{\frac{\partial}{\partial V_{\mathrm{G}}}(W)}\right|_{V_{\mathbf{G}}=V_{\mathrm{TC}}}
$$

où $L_{\mathrm{D}}$ est la longueur de Debye extrinsèque, soit encore

$$
\left|V_{\mathrm{C}}-V_{\mathrm{TC}}\right| \simeq 2 \sqrt{\frac{k T}{q}\left(V_{\mathrm{bi}}-V_{\mathrm{TC}}\right)} .
$$

4.2 VÉRIFICATIONS EXPÉRIMENTALES. - 4.2.1 Caractéristiques courant-tension. - Quatre types de vérifications des relations précédentes peuvent être effectués à partir de la mesure des caractéristiques de courant drain en fonction des tensions grille et substrat, à faible tension drain-source :

(i) Dans le plan $I_{\mathrm{D}}\left(V_{\mathrm{G}}\right)$, en considérant la tension substrat comme paramètre, l'analyse théorique montre que les caractéristiques sont confondues tant que $V_{\text {su }}$ est supérieur à $V_{\text {sul }}$ (7).

Ensuite elles se translatent selon l'axe des courants drain d'une quantité dépendant de la polarisation de substrat comme le montre l'expression (9) du courant écrite sous la forme :

$$
\begin{aligned}
\left.I_{\mathrm{D}}\right|_{V_{\mathrm{D} \rightarrow 0}}=\mu_{0} \frac{\mathrm{Z}}{L} q N_{\mathrm{D}} V_{\mathrm{D}} \times \\
\times\left[d+\frac{\sigma_{\mathrm{ss}}}{q N_{\mathrm{D}}}-\sqrt{\frac{2 \varepsilon_{\mathrm{s}} \varepsilon_{0}}{q N_{\mathrm{D}}} \frac{N_{\mathrm{t}}}{N_{\mathrm{D}}}\left(\varphi_{\mathrm{D}}-V_{\mathrm{su}}\right)}-\right. \\
\left.-\sqrt{\frac{2 \varepsilon_{0} \varepsilon_{\mathrm{s}}}{q N_{\mathrm{D}}}\left(V_{\mathrm{bi}}-V_{\mathrm{G}}\right)}\right]
\end{aligned}
$$

Ce résultat est conforme à l'observation expérimentale (Fig. 4). Il fait en particulier apparaître qu'il y a sensiblement conservation de la transconductance - à tension grille fixée - lorsque la tension de substrat varie, à condition, bien entendu que le transistor reste dans l'état conducteur.

(ii) Afin de mieux préciser ces points, on peut utiliser premièrement les résultats expérimentaux de la figure 5 qui représente les variations du courant de drain $I_{D O}$ en fonction de la tension de substrat pour une tension grille-source nulle. Pour les valeurs de la tension de substrat supérieures à $V_{\text {sul }}$ le courant reste constant et égal à la valeur notée $I_{\mathrm{DL}}$. Ensuite on observe une décroissance du courant. Afin de vérifier le bien-fondé de la relation théorique suivante, déduite de (7) et (16) :

$$
\begin{aligned}
& \left.\left(I_{\mathrm{DL}}-I_{\mathrm{DO}}\right)\right|_{\substack{V_{\mathrm{su}}<V_{\mathrm{sul}} \\
V_{\mathrm{D} \rightarrow 0}}}=q N_{\mathrm{D}} \mu_{0} V_{\mathrm{D}} \frac{Z}{L} \times \\
& \times\left[\sqrt{\frac{2 \varepsilon_{0} \varepsilon_{\mathrm{s}}}{q N_{\mathrm{D}}} \frac{N_{\mathrm{t}}}{N_{\mathrm{D}}}\left(\varphi_{\mathrm{D}}-V_{\mathrm{su}}\right)}-\frac{\sigma_{\mathrm{ss}}}{q N_{\mathrm{D}}}\right] .
\end{aligned}
$$

on a reporté sur la figure 9 les variations de $\left(I_{\mathrm{DL}}-I_{\mathrm{DO}}\right)$, obtenues expérimentalement, en fonction de la racine carrée du potentiel $\varphi_{\mathrm{D}}-V_{\mathrm{su}}$. La tension

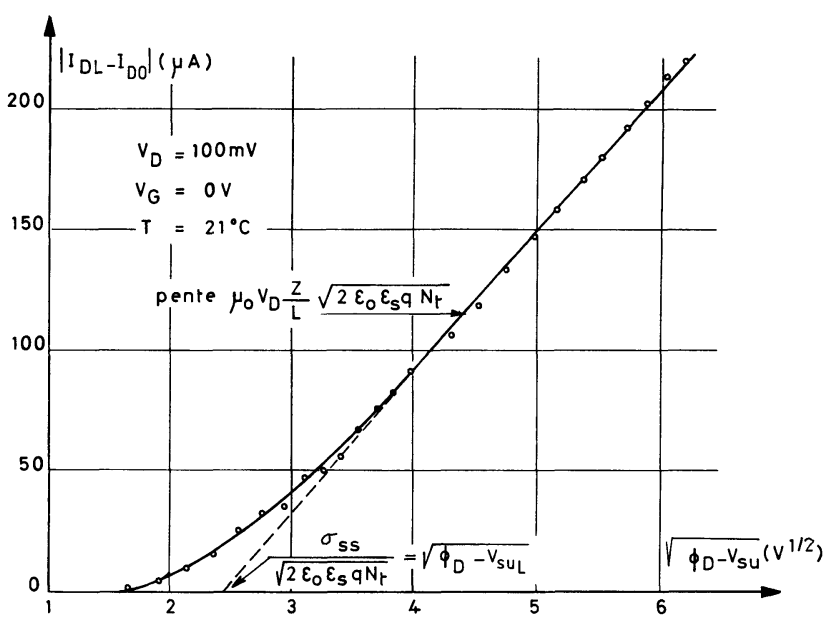

FIG. 9. - Variations expérimentales de la différence des courants $I_{\mathrm{DL}}-I_{\mathrm{Do}}$ en fonction de la racine carrée du potentiel $\left(\varphi_{\mathrm{D}}-V_{\mathrm{su}}\right)$.

Détermination des quantités $\mu_{0} \frac{Z}{L} \sqrt{2 \varepsilon_{\mathrm{s}} \varepsilon_{0} q N_{\mathrm{t}}}$ et $\frac{\sigma_{\mathrm{ss}}}{2 \varepsilon_{\mathrm{s}} \varepsilon_{0} q N_{\mathrm{t}}}$.

[Experimental variations of the $I_{\mathrm{DL}}-I_{\mathrm{Do}}$ current difference as a function of the square-root of the $\left(\varphi_{\mathrm{D}}-V_{\mathrm{su}}\right)$ voltage.]

$\varphi_{\mathrm{D}}$ est prise égale à $0,7 \mathrm{~V}$, moitié de la bande interdite de l'arséniure de gallium. Ces variations sont linéaires dès que le potentiel $V_{\text {su }}$ est inférieur à $V_{\text {sul }}$. Ce tracé fournit :

- par mesure de la pente, la quantité

$$
\mu_{0} V_{\mathrm{D}} \frac{Z}{L} \sqrt{2 \varepsilon_{\mathrm{s}} \varepsilon_{0} q N_{\mathrm{t}}}
$$

- par mesure de l'abscisse à l'origine la quantité $\sqrt{\varphi_{\mathrm{D}}-V_{\text {sul }}}$ soit encore $\frac{\sigma_{\mathrm{ss}}}{\sqrt{2 \varepsilon_{\mathrm{s}} \varepsilon_{0} q N_{\mathrm{t}}}}$;

- par mesure de l'ordonnée à l'origine la quantité $-\sigma_{\mathrm{ss}} \frac{Z}{L} \mu_{0} V_{\mathbf{D}}$

(iii) Toujours dans le cadre de vérification de la dépendance du courant en fonction des tensions de grille et de substrat, la figure 10 représente les variations expérimentales du courant de drain en fonction de la racine carrée du potentiel $V_{\mathrm{bi}}-V_{\mathrm{G}}$. Le potentiel $V_{\text {bi }}$ comme nous le verrons par ailleurs, 


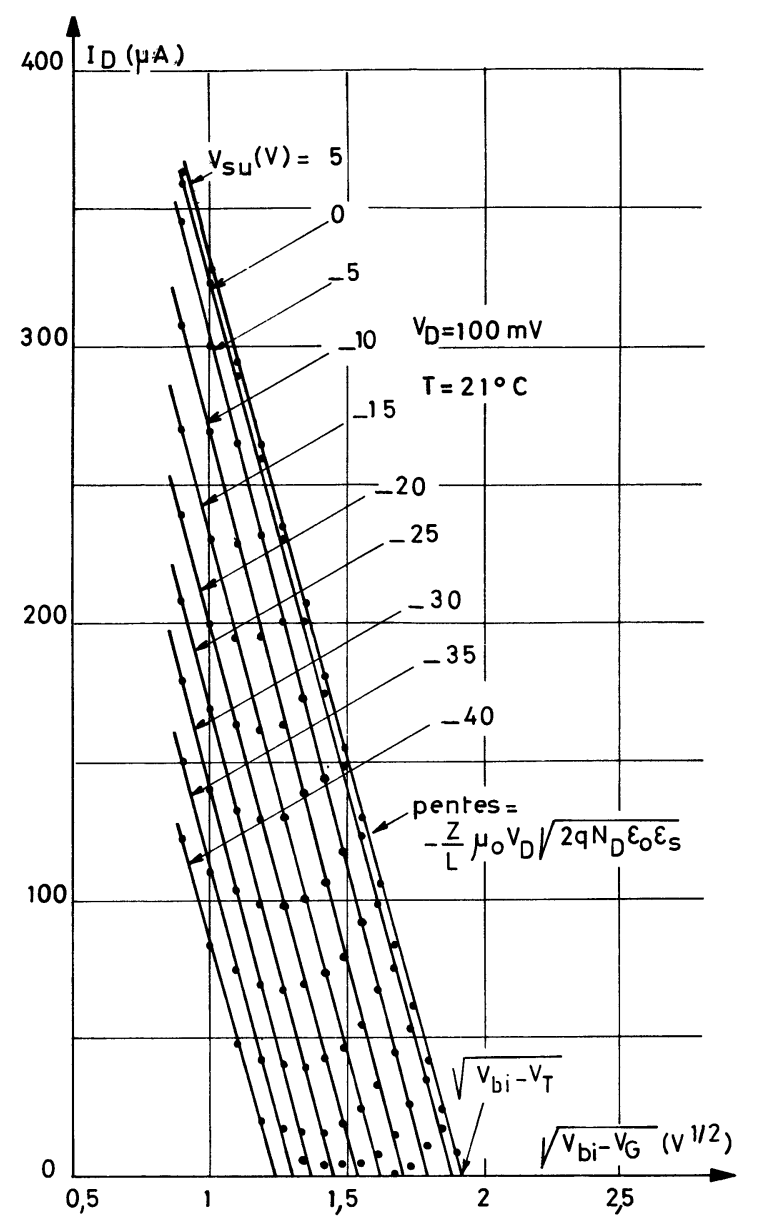

FIG. 10. - Variations expérimentales du courant de drain $I_{\mathrm{D}}$ en fonction de la racine carrée du potentiel $\left(V_{\mathbf{b i}}-V_{\mathrm{G}}\right)$, pour diverses valeurs de la tension de substrat $V_{\mathrm{su}}$. Application à la détermination des tensions de seuil $V_{\mathrm{T}}\left(V_{\mathrm{su}}\right)$ et de la quantité $\mu_{0} \frac{Z}{L} \sqrt{2 \varepsilon_{\mathrm{s}} \varepsilon_{0} q N_{\mathrm{D}}}$. Structure THOMSON SCHOT-3, T317 No 2.

[Experimental variations of the drain current as a function of the square-root of the $\left(V_{\mathrm{bi}}-V_{\mathrm{G}}\right)$ voltage.]

est déduit des courbes capacité-tension. Les variations sont linéaires conformément aux relations (7) et (16). On obtient :

- par mesure de la pente qui est constante la quantité $-\frac{Z}{L} \mu_{0} V_{\mathrm{D}} \sqrt{2 q N_{\mathrm{D}} \varepsilon_{0} \varepsilon_{\mathrm{s}}}$

- par mesure de l'abscisse à l'origine, la quantité $\sqrt{V_{\mathrm{bi}}-V_{\mathrm{T}}}$ en fonction du paramètre $V_{\mathrm{su}}$.

(iiii) Pour être complet, après avoir déterminé expérimentalement cette quantité $\sqrt{V_{\mathrm{bi}}-V_{\mathrm{T}}}$ pour chaque valeur de la polarisation du substrat, on trace ses variations en fonction de la racine carrée du potentiel $\varphi_{\mathrm{D}}-V_{\text {su }}$ (Fig. 11).

La caractéristique obtenue est composée de deux portions de droites, une horizontale correspondant à $\sqrt{\varphi_{\mathrm{D}}-V_{\mathrm{su}}}<\sqrt{\varphi_{\mathrm{D}}-V_{\text {sul }}}$, et une droite à pente négative correspondant à l'inégalité opposée.

Compte tenu des expressions théoriques du seuil, (8) et (10), l'ordonnée de la partie horizontale repré-

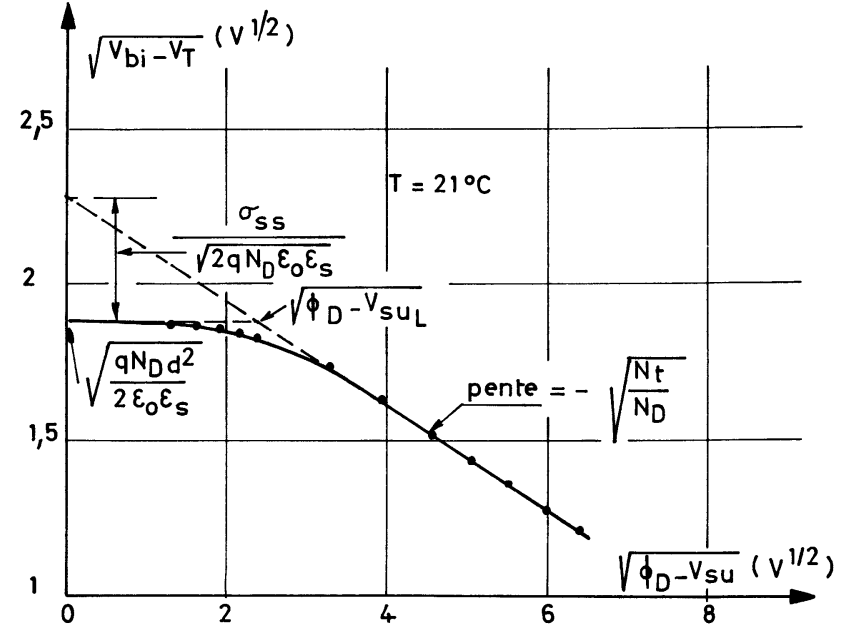

FIG. 11. - Variations expérimentales de la racine carrée du potentiel $\left(V_{\mathrm{bi}}-V_{\mathrm{T}}\right)$ en fonction de la racine carrée du potentiel $\left(\varphi_{\mathrm{D}}-V_{\mathrm{su}}\right)$. Application à la détermination des quantités :

$\sqrt{\frac{N_{\mathrm{t}}}{N_{\mathrm{D}}}}, \sqrt{\varphi_{\mathrm{D}}-V_{\mathrm{sul}}}, \sqrt{\frac{q N_{\mathrm{D}} d^{2}}{2 \varepsilon_{0} \varepsilon_{\mathrm{s}}}}$ et $\frac{\sigma_{\mathrm{ss}}}{\sqrt{2 q N_{\mathrm{D}} \varepsilon_{0} \varepsilon_{\mathrm{s}}}}$. Structure

THOMSON SCHOT-3, T317 No 2 .

[Experimental variations of the square-root of $\left(V_{\mathrm{b}_{1}}-V_{\mathrm{T}}\right)$ as a function of the square-root of $\left(\varphi_{\mathrm{D}}-V_{\mathrm{su}}\right)$.]

sente la quantité $\sqrt{\frac{q N_{\mathrm{D}} d^{2}}{2 \varepsilon_{0} \varepsilon_{\mathrm{s}}}}$, par ailleurs la pente de l'autre portion linéaire est égale à $-\sqrt{\frac{N_{\mathrm{t}}}{N_{\mathrm{D}}}}$ et son ordonnée à l'origine obtenue par extrapolation fournit la quantité $\sqrt{\frac{q N_{\mathrm{D}} d^{2}}{2 \varepsilon_{0} \varepsilon_{\mathrm{s}}}}+\frac{\sigma_{\mathrm{ss}}}{\sqrt{2 q N_{\mathrm{D}} \varepsilon_{0} \varepsilon_{\mathrm{s}}}}$.

4.2.2 Caractéristiques capacité-tension. - Sur la figure 12 on a reporté les variations de l'inverse du carré de la capacité grille-(drain, source) en fonction de la tension de grille. On observe une partie linéaire, indépendante de la tension de substrat, qui obéit à la relation :

$$
\frac{1}{C^{2}}=\frac{2\left(V_{\mathrm{bi}}-V_{\mathrm{G}}\right)}{q \varepsilon_{0} \varepsilon_{\mathrm{s}} N_{\mathrm{D}}} \cdot \frac{1}{(Z L)^{2}} .
$$

De la mesure de la pente on déduit la quantité $\frac{2}{q \varepsilon_{0} \varepsilon_{\mathrm{s}} Z^{2} L^{2} N_{\mathrm{D}}}$ et par détermination de l'ordonnée à l'origine on mesure le potentiel $V_{\mathrm{bi}}$. Par ailleurs le réseau de caractéristiques obtenues présente ensuite, des portions très rapidement croissantes qui coupent la partie linéaire pour une valeur de tension grille $V_{\mathrm{TI}}$ très proche de la tension de seuil $V_{\mathrm{T}}$. Il est ainsi possible de déterminer la caractéristique de tension de seuil en fonction de la tension substrat et, par une méthode identique à celle décrite au paragraphe 4.2.1(iii), de tracer la courbe $\sqrt{V_{\mathrm{bi}}-V_{\mathrm{TI}}}$ 


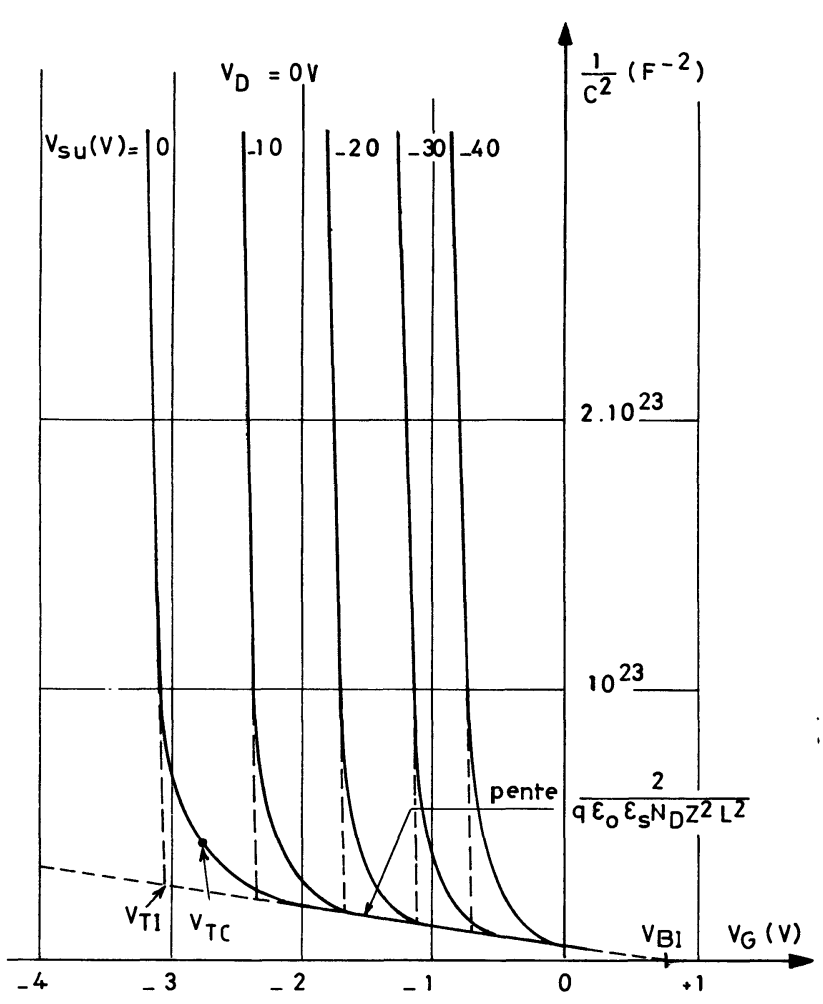

FIG. 12. - Variations expérimentales de l'inverse du carré de la capacité de grille en fonction de la tension de grille. Application à la détermination des tensions de seuil $V_{\mathrm{TI}}\left(V_{\mathrm{su}}\right)$ et de la quantité $\frac{2}{q \varepsilon_{0} \varepsilon_{\mathrm{s}} Z^{2} L^{2} N_{\mathrm{D}}}$. Structure THOMSON SCHOT-3, T317 No 1.

[Experimental variations of the value of the reverse of the square of the gate capacitance as a function of the gate voltage.]

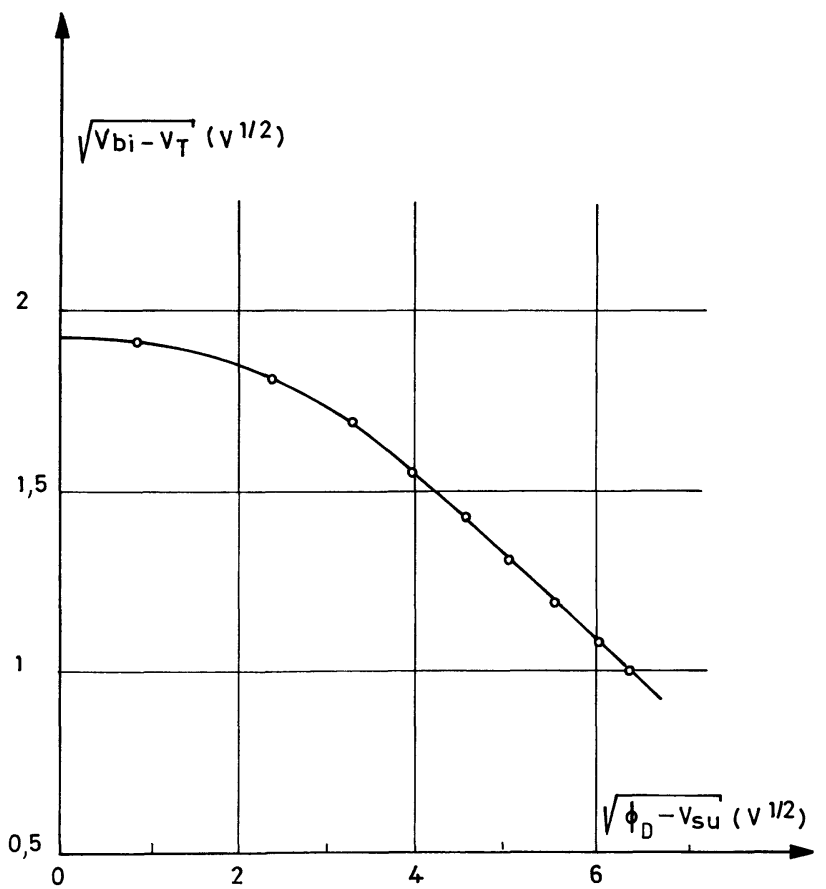

FIG. 13. - Variations expérimentales de la racine carrée du potentiel $\left(V_{\mathrm{bi}}-V_{\mathrm{T}}\right)$ en fonction de la racine carrée du potentiel $\left(\varphi_{\mathrm{D}}-V_{\mathrm{su}}\right)$. Résultats déduits des caractéristiques capacité-tension Structure THOMSON SCHOT-3, T317 No 1.

[Experimental variations of the square-root of $\left(V_{\mathrm{bi}}-V_{\mathrm{TI}}\right)$ as a function of the square-root of $\left(\varphi_{D}-V_{\text {su }}\right)$. These results are obtained by the voltage-capacitance method.] fonction de $\sqrt{\varphi_{\mathrm{D}}-V_{\text {su }}}$ pour obtenir respectivement les quantités $\frac{q N_{\mathrm{D}} d^{2}}{2 \varepsilon_{0} \varepsilon_{\mathrm{s}}}, \sqrt{\frac{N_{\mathrm{t}}}{N_{\mathrm{D}}}}$ et $\frac{\sigma_{\mathrm{ss}}}{\sqrt{2 q N_{\mathrm{D}} \varepsilon_{0} \varepsilon_{\mathrm{s}}}}$ (Fig. 13).

4.2.3 Application à la détermination des paramètres physiques des couches. - Il ressort premièrement de l'analyse précédente que, par mesure des caractéristiques capacité-tension, avec source et drain court-circuités, on peut déterminer le dopage $N_{\mathrm{D}}$ de la couche semiconductrice, la hauteur de barrière $V_{\mathrm{bi}}$, l'épaisseur $d$ de la couche, la densité de centres profonds $N_{\mathrm{t}}$ et la charge superficielle $\sigma_{\mathrm{ss}}$.

Deuxièmement, les quantités

$$
N_{\mathrm{D}} d^{2}, \sqrt{N_{\mathrm{D}}} \cdot \mu_{0}, \frac{\sigma_{\mathrm{ss}}}{\sqrt{N_{\mathrm{D}}}}, \frac{N_{\mathrm{t}}}{N_{\mathrm{D}}}
$$

peuvent être aussi déduites des caractéristiques courant-tension à faible tension drain-source. On vérifie que les déterminations effectuées par les deux méthodes se recoupent.

Ainsi il apparaît comme indispensable d'utiliser les deux méthodes pour obtenir tous les paramètres de la couche, à savoir : $N_{\mathrm{D}}, V_{\mathrm{bi}}, d, N_{\mathrm{t}}, \mu_{0}, \sigma_{\mathrm{ss}}$. Un exemple de valeurs de ces paramètres pour des couches ultraminces obtenues par cracking de composés organo-métalliques sous pression réduite [6] (série THOMSON CSF 310-6) sont les suivantes à la température de $300 \mathrm{~K}$ :

$$
\begin{aligned}
& \begin{array}{cccccc}
\begin{array}{ccc}
N_{\mathrm{D}} \\
\left(\mathrm{cm}^{-3}\right)
\end{array} & \begin{array}{c}
V_{\mathrm{bi}} \\
(\mathrm{V})
\end{array} & \begin{array}{c}
d \\
(\mu \mathrm{m})
\end{array} & \begin{array}{c}
N_{\mathrm{t}} \\
\left(\mathrm{cm}^{-3}\right)
\end{array} & \begin{array}{c}
\mu_{0} \\
\left(\mathrm{~cm}^{2} \cdot \mathrm{V}^{-1} \cdot \mathrm{s}^{-1}\right)
\end{array} & \begin{array}{c}
\sigma_{\mathrm{ss}} \\
\left(\mathrm{Cb} \cdot \mathrm{m}^{-2}\right)
\end{array}
\end{array} \\
& \begin{array}{cccccc}
7,6 \times 10^{16} & \overline{0,75} & \overline{0,26} & 1, \overline{9} \times 10^{15} & 3 \overline{900} & 6 \times 10^{-4}
\end{array}
\end{aligned}
$$

Le potentiel $V_{\mathrm{bi}}$ est relatif à une grille de titane et la densité de charges fixes d'interface $N_{\mathrm{ss}}$, associée à la charge $\sigma_{\mathrm{ss}}$, est de $3,8 \times 10^{11} \mathrm{~cm}^{-2}$.

On doit enfin rappeler que les caractéristiques capacité-tension ne permettent de mesurer, en tout état de cause, que le profil de porteurs libres $n(x)$ et, que l'exploitation, généralement utilisée, de ces caractéristiques (structures à grille Schottky ou à double goutte de mercure) [10] pour obtenir le profil de dopage et l'épaisseur métallurgique de la couche n'est qu'un artifice [15]. En particulier, la chute de capacité pour les tensions de grille élevées, n'est pas toujours associée à une non-uniformité de dopage mais peut être due à l'interaction de deux zones de charges d'espace dépeuplées, particulièrement lorsque le potentiel électrostatique du substrat n'est pas imposé (cf. Fig. 3).

5. Conclusion. - L'influence de la polarisation du substrat semi-isolant sur les caractéristiques de courant et de capacité de grille en fonction des tensions de grille et de drain, a été étudiée expérimentalement sur des transistors à effet de champ à l'arseniure 
de gallium à grille métallique. Les évolutions observées ont été associées à la formation d'une charge d'espace dans la couche épitaxiale $\mathrm{N}$, conductrice, du côté de l'interface. La nature et les propriétés de cette charge d'espace dépendent de l'amplitude de la tension de substrat : elle peut être constituée soit par une zone d'accumulation en électrons, soit par une zone de dépeuplement. Il a été mis en évidence que le substrat semi-isolant présente en régime statique un comportement de type diélectrique ou de type semiconducteur $\mathrm{P}$, selon la polarité et l'amplitude de la tension de substrat. On a enfin montré qu'il existe, à l'interface couche épitaxiale-substrat semi-isolant, une charge fixe superficielle, positive, qui impose un seuil au-delà duquel l'effet de tension substrat devient sensible.

D'un point de vue quantitatif, une formulation a été proposée pour décrire les évolutions du courant drain, à faible tension drain-source, et de la capacité de grille. Cette analyse a permis de définir des méthodes de détermination des paramètres physiques de la couche $\mathrm{N}$ tels que l'épaisseur, le dopage, la tension de diffusion de la barrière Schottky et la mobilité, et des paramètres caractéristiques de l'interface tels que la densité superficielle de charges fixes et la densité de centres profonds.

En pratique il apparaît que, abstraction faite de la mobilité, tous ces paramètres peuvent être obtenus par une mesure de capacité-tension. Ces résultats indiquent que les méthodes classiques, non destructives et ne nécessitant pas de traitement technologique des couches, telle que la technique de la double goutte de mercure, ne sont pas, dans certains cas, utilisables sans ambiguïté pour la détermination du profil de dopage de la couche. Cependant elles peuvent être transposées pour effectuer une caractérisation complète de la couche et de l'interface à condition d'élaborer sur les structures à tester un contact sur le substrat.

Enfin, d'une manière plus générale, la mise en évidence de l'influence de la polarisation du substrat, montre que le transistor à effet de champ à l'arseniure de gallium doit être considéré a priori comme un dispositif à quatre électrodes. Des recherches en cours permettront de préciser l'influence de cette polarisation de substrat sur les caractéristiques dynamiques hyperfréquences telles que le gain en puissance et le facteur de bruit.

\section{Bibliographie}

[1] Rossel, P., Tranduc, H., Graffeuil, J., Azizi, C., Revue Phys. Appl. 12 (1977) 1679.

[2] Barrera, J., The importance of Substrate Properties on GaAs FET Performance. 5th Biennal Cornell Electrical Conference. Sec. II 3 (1975) 135.

[3] Nozaki, T., Ogawa, M., Terao, H., Watanabe, H., MultiLayer Epitaxial Technology for the Schottky Barrier GaAs FET. Gallium Arsenide and Related Compounds. Inst. Phys. Conf. Ser. no 24 (1975) ch 2, 46.

[4] Yokohama, N., Shibatomi, A., Ohkawa, S., Fukuta, M., Hishikawa, H., Electrical Properties of the Interface between an N.GaAs Epitaxial Layer and a Cr-Doped Substrate. Gallium Arsenide and Related Compounds. Inst. Phys. Conf. Ser., no 33 h (1977) ch 4, 201.

[5] HoOper, W. W., CaIRns, B. R., Feasibility of a Gallium Arsenide Schottky Barrier Gate Field-Effect transistor. Technical Report AFAL-TR-69 30 (1969) 28.

[6] Duchemin, J. P., Bonnet, M., Huyghe, D., Revue Technique Thomson CSF 9 (1977) 685.

[7] GloANEC, M., Conception et caractérisation d'un dispositif Gunn triode. Diplome CNAM (1975) 56.

[8] GloANEC, M., Etude de la faisabilité de triode planar à effet Gunn utilisables en électronique rapide. Compte rendu final - Convention DGRST CCM 75-7-0668 (1976) 15.
[9] Yamashita, A., Yamaguchi, M., Aoki, T., Japan. J. Appl. Phys. 12 (1973) 1267.

[10] BINET, M., Electron. Lett. 11 (1975) 580.

[11] Tanimoto, M., Suzuki, K., IтoH, T., Iкoma, T., Yanal, H., KaufmanN, L. M. F., Nievendick, W., Heime, K., 6th European Solid State Device Research Conference, München (1976) secc. B4.

[12] Lehovec, K., Zuleeg, R., Inst. Phys. Conf. Ser. no 24 (1975) ch $5,292$.

[13] Lehovec, K., Appl. Phys. Lett. 26 (1975) 82.

[14] Azizi, C., Rossel, P., Tranduc, H., Graffeuil, J., C.R. Hebd. Séan. Acad. Sci. B 285 (1977) 145

[15] Kennedy, D. P., O'Brien, R. R., IBM J. Res. Develop. 13 (1969) 212.

[16] Huber, A., Moulin, M., Caractérisation des matériaux et technologies semiconducteurs, Grenoble 27-29 sept. 1972, p. 447.

[17] Huber, A. M., Morillot, G., Linh, N. T., Debrun, J. L., VAlladon, M., Nucl. Instrum. Methods. 149 (1978) 543.

[18] Houng, Y., Preparation and Properties of Semi-Insulating Liquid Phase Epitaxial GaAs for Application to FET Devices. Ph. D. Thesis, Stanford University (1977) ch. 5. 